\title{
A Kind of FEM Dummy Material Parameter Curve Optimization Method Based on Vehicle Collision Simulation
}

\author{
Kai Ma ${ }^{1,2}$, Lei $\operatorname{Yan}^{1, *}$, Tao Xu $^{1} \&$ Jidong Gao ${ }^{2}$ \\ 1. College of mechanical Science and Engineering, Jilin University, Changchun, China 130022; \\ 2. Automotive Engineering Research Institute, China Automotive Technology \& Research \\ Center,TianJing, China 300300
}

Keywords: Vehicle collision;FE dummy;Parametric curve optimization;Parameter optimization

Abstract. Based on full-size vehicle collision test, a new finite element dummy model(FEM dummy) was built in this paper, at the same time the corresponding dummy's material parameter curve optimization method was proposed. For the result of dummy simulation being in keep with that of full scale vehicle collision experiments, it was necessary to scientifically adjust the dummy's structural material parameters and parameters curve. Besides, optimized design of parameters curve was a more complex work. Therefore proposing a method of the curve approximate optimization to meet the design requirements was necessary. First, according to the variation of the curve, we cut it into segments, and redefined them as parameters. Then, using the parameter optimization method, the important parameters was selected which significantly affected the results of the simulation. Finally, using that method obtained the curve which made it consistent with the full-scale vehicle collision test results. In the paper, positive collision chest pressure test of a new FEM dummy as an example, to make sure simulation dummy' chest pressure peak and spring rate and so on were consistent with full-size vehicle collision dummy data, Use this method to optimization design someone material parameter curve. The result proved that the method could be used to improve the new FEM dummy to meet the results of the full-scale vehicle collision test.

\section{Introduction}

Vehicle crash simulation is an important link in the process of product development, It is particularly important as a dummy evaluation index ${ }^{[1]}$, From 80 s onwards, the industrial world and the academic circles have begun to establish the simulation model. now, more widely used is the the III 50th Hybrid finite element model established by the United States LSTC company and polyhedron model established by TNO company,But these two models have their own shortcomings[2]. When LSTC company is in the continuous improvement of the performance of the model and the accuracy of the performance, it s computing time increases; TNO company's model is not the real entity finite element model although the calculation speed is fast, And they are all simulated by the human characteristics of the western countries ${ }^{[3-8]}$. The research for the dummy in our country started late, Chen Shuang, Yuan Zhong Fan etc ${ }^{[9]}$,established The Chinese 95\% dummy based on the human body mechanics and finite element analysis method, It basically realized Simulation of mechanical structure and similarity of mechanical properties of crash dummy. Cai Zhihua, LAN Fengchong etc[10], established the finite element model of human thorax based on the damage of vehicle collision, and use it to predict and evaluate the biomechanical response and damage mechanism of the chest under the impact environment.But the model used in the test data is a western data, can not fully reflect the Chinese people's biomechanical response.

Based on a new domestic finite element simulation dummy model, This paper aims to solve the 
problem of low calculation accuracy of the dummy simulation model. In order to make the simulation effect of dummy in accordance with real experiment results, The general need to adjust the structure of the main material parameters and dummy parameter curve. In this paper, a method to satisfy the requirements of curve approximation optimization design method is presented. In order to illustrate the application of the method, This paper takes a new dummy FE model Frontal collision thoracic pressure test as an example, Using this method, a material parameter curve is designed. Results showed, This method can be used to improve the needs of a new dummy FE model to meet the vehicle crash simulation accuracy.

\section{Objective curve optimization method}

In engineering, a lot of optimization parameters and optimization goals are curve. And most of these curves are obtained through experiment and simulation. Each curve cannot be expressed explicitly, It is more difficult to use a functional representation of the relationship between them. Although, the current parameter fitting technology has been very developed, But in terms of engineering problems, It is too cumbersome. So this paper puts forward the optimization method of curve to curve for engineering application.

First of all, from the perspective of parameter sensitivity, The parametric curve can be divided into several segments, The slope and length of each segment have different sensitivity to the result of the target. From the point of view of the optimization goal, The target result may be the peak value of the target curve、Peak position or slope, etc. These can be directly observed and described by a visual indicator.

In this way, the parametric curve can be expressed in the form of parameterization, For example, each line segment length and slope, And the target curve can be parameterized, Such as peak value and slope, etc. As a result, the optimization problem of the curve to curve can be described as a multi objective and multi parameter optimization problem.

Here is a brief introduction to the multi-objective optimization method used in this paper[10].

Iterative calculation step by step. Specific steps are as follows:

Step one: Set a minimum $\Delta x$, That is initial interval radius, using difference method to find the sensitivity of function.

$$
d f_{k} / d x_{i}=\frac{f_{k}\left(x_{1}, x_{2}, \mathrm{~L}, x_{i}+\Delta x, \mathrm{~L}, x_{n}\right)-f_{k}(X)}{\Delta x}
$$

Step two:Calculate

$$
\frac{d f_{j} / d x_{i}}{d f_{k} / d x_{i}} \quad k, j=1,2, \mathrm{~L} m \quad i=1,2, \mathrm{~L}, n
$$

Step three: The following equations are used to solve $\lambda_{1}, \lambda_{2}, \mathrm{~L}, \lambda_{m}$. 


$$
\left[\begin{array}{cccc}
\sum_{i=1}^{n} \frac{d f_{1} / d x_{i}}{d f_{1} / d x_{i}} & \sum_{i=1}^{n} \frac{d f_{1} / d x_{i}}{d f_{2} / d x_{i}} & \mathrm{~L} & \sum_{i=1}^{n} \frac{d f_{1} / d x_{i}}{d f_{m} / d x_{i}} \\
\sum_{i=1}^{n} \frac{d f_{2} / d x_{i}}{d f_{1} / d x_{i}} & \sum_{i=1}^{n} \frac{d f_{2} / d x_{i}}{d f_{2} / d x_{i}} & \mathrm{~L} & \sum_{i=1}^{n} \frac{d f_{2} / d x_{i}}{d f_{m} / d x_{i}} \\
\mathrm{M} & \mathrm{O} & \\
\sum_{i=1}^{n} \frac{d f_{m} / d x_{i}}{d f_{1} / d x_{i}} & \sum_{i=1}^{n} \frac{d f_{m} / d x_{i}}{d f_{2} / d x_{i}} & \mathrm{~L} & \sum_{i=1}^{n} \frac{d f_{m} / d x_{i}}{d f_{m} / d x_{i}}
\end{array}\right] \cdot\left[\begin{array}{c}
\lambda_{1} \\
\lambda_{2} \\
\mathrm{M} \\
\lambda_{m}
\end{array}\right]^{T}=\left[\begin{array}{c}
\Delta f_{1}(\mathbf{X}) \\
\Delta f_{2}(\mathbf{X}) \\
\mathrm{M} \\
\Delta f_{m}(\mathbf{x})
\end{array}\right] \text { (3) }
$$

Step four:

$$
\Delta x_{i}=\lambda_{1} \frac{d f_{1}}{d x_{i}}+\lambda_{2} \frac{d f_{2}}{d x_{i}}+\lambda_{3} \frac{d f_{3}}{d x_{i}}
$$

Step five:

$$
\Delta f_{k}(\mathbf{X})=f_{k}(\mathbf{X}+\Delta \mathbf{X})-f_{k}(\mathbf{X})
$$

If $\left\|\Delta f_{k}(\mathbf{X})-\Delta f_{k \max }(\mathbf{X})\right\|_{f} \leq \varepsilon$, stop calculate; otherwise,order

$$
d f_{k} / d x_{i}=\frac{f_{k}\left(x_{1}, x_{2}, \mathrm{~L}, x_{i}+\Delta x_{i}+\Delta x, \mathrm{~L}, x_{n}\right)-f_{k}(X)}{\Delta x}
$$

Back to the second step.

Here, $\varepsilon$ is a given threshold.

\section{Example}

Take frontal impact chest compression test of a new type of dummy Finite Element Model as an example. It's model is shown in Figure 2. Reference frontial impact test in C-NCAP regulations and chest calibration method in NHTSA CFR Part 572 issued by United States National Highway Safety Administration [14-16]. In this paper, a simplified frontal collision test is established. Build a simplified seat model, place dummy FE model, establish a rectangular solid pendulum with a mass of $10 \mathrm{~kg}$ and with a impacted area of $70 \mathrm{~mm} * 90 \mathrm{~mm}$, With the speed of $5.77 \mathrm{~m} / \mathrm{s}$ impact dummy FE model, After calculate with LS-DYNA software, check the dummy's chest pressure curve. The calculated chest pressure curve is shown in Figure 4, among, $\mathrm{K}_{1^{--}}$The curve segment between the initial curve and the peak value of the thoracic pressure, calculate the approximate descending slope $\mathrm{K}_{1}$. By the same way to calculate $\mathrm{K}_{2}$, The curve segment between the peak value and the Rebound stage of the thoracic pressure, calculate the approximate Rebound slope $K_{2} . D_{\max }$ represents the peak of the thoracic pressure curve. The initial value of the thoracic pressure curve is shown in Table 1 and figure 3.

Select a dummy chest material stress strain curve, as shown in Figure 4. The parametric curve is decomposed into 4 line segments. There are 8 characteristic parameters which reflect the characteristic of the parametric curve, using $\mathrm{m}_{1}, \mathrm{~m}_{2}, \mathrm{~m}_{3}, \mathrm{~m}_{4}$ to express the slope of line segment $\mathrm{AB}$, $\mathrm{BC}, \mathrm{CD}$ and $\mathrm{DE}$ of line segment, using $\mathrm{N}_{1}, \mathrm{~N}_{2}, \mathrm{~N}_{3}, \mathrm{~N}_{4}$ express each line segment in $\mathrm{X}$ axis projection.,the initial values of the parameters are shown in Table 2. These 8 parameters are used as optimization parameters to optimize the slope and peak of the thoracic pressure curve.

Design parameters: $m_{1}, m_{2}, m_{3}, m_{4}, n_{1}, n_{2}, n_{3}, n_{4}$ 
Design objective:

1.The value of thoracic pressure peak $D_{\max }$ is $30 \mathrm{~mm}$

2.The descending slope and rebound slope of thoracic pressure curve is $K_{1}$ and $K_{2}$.

$$
\begin{aligned}
& D_{\max }\left(m_{1}, m_{2}, m_{3}, m_{4}, n_{1}, n_{2}, n_{3}, n_{4}\right)=30 \\
& K_{1}\left(m_{1}, m_{2}, m_{3}, m_{4}, n_{1}, n_{2}, n_{3}, n_{4}\right)=-2.5 \\
& K_{2}\left(m_{1}, m_{2}, m_{3}, m_{4}, n_{1}, n_{2}, n_{3}, n_{4}\right)=2
\end{aligned}
$$

In a numerical example, the curve optimization method is introduced in this paper, the optimization was carried out according to the optimized target value, finally get the optimal solution, as shown in Figure 1 and table 3, The stress-strain curve after optimization is shown in Figure 2, the main characteristic values of stress strain curves are shown in Table 4. From the optimization results, it's optimal solution is very close to the design goal, it shows that the method can meet the requirements of optimization.

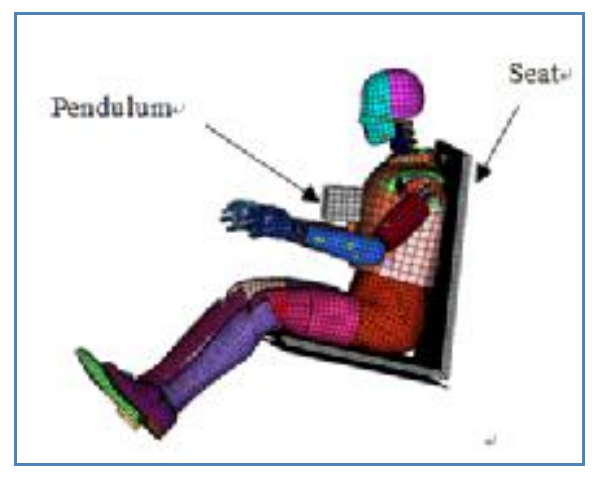

Fig. 1 The frontal collision thoracic pressure test

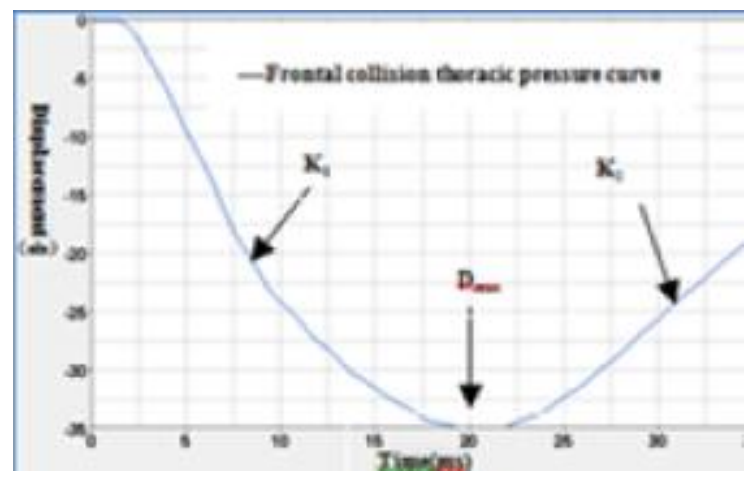

Fig. 2 The frontal collision thoracic pressure curve before optimization

Table 2 Characteristic parameter values of stress and strain curve before optimization

Table 1 Evaluation indexes of thoracic pressure curve

\begin{tabular}{cl|c|c}
$\begin{array}{c}\text { Evaluation } \\
\text { indexes }\end{array}$ & $\mathrm{D}_{\max } /(\mathrm{mm})$ & $\mathrm{K}_{1}$ & $\mathrm{~K}_{2}$ \\
Value & 35.110 & -3.311 & 1.169
\end{tabular}

\begin{tabular}{ccccc}
\hline Parameter & $\mathrm{m}_{1}$ & $\mathrm{~m}_{2}$ & $\mathrm{~m}_{3}$ & $\mathrm{~m}_{4}$ \\
Value & 0.36 & 2.025 & 8.03 & 35.72 \\
Paremeter & $\mathrm{n}_{1}$ & $\mathrm{n}_{2}$ & $\mathrm{n}_{3}$ & $\mathrm{n}_{4}$ \\
Value & 0.6 & 0.2 & 0.1 & 0.1
\end{tabular}

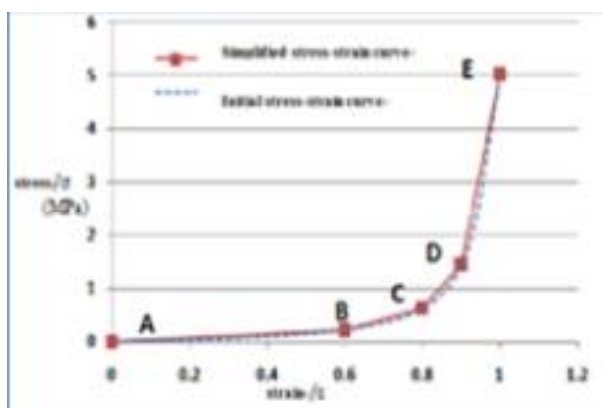

Fig. 3 The stress-strain curve of the material before optimization

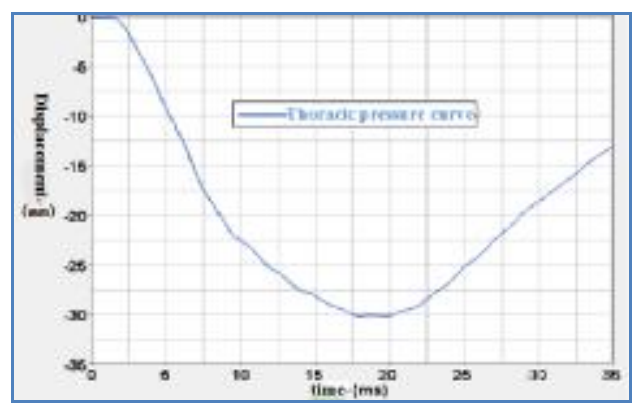

Fig. 4 The frontal collision thoracic pressure curve after optimization 


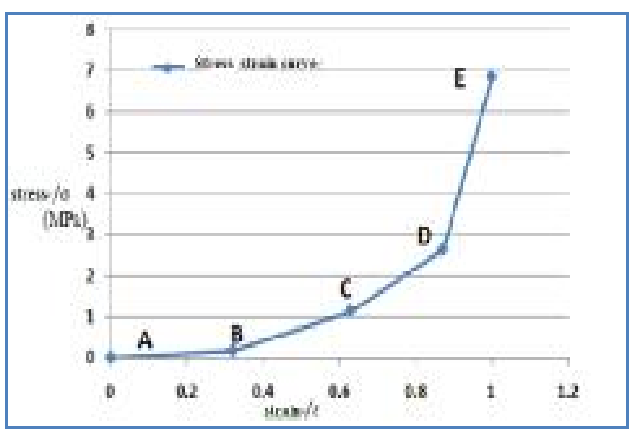

Fig. 5 The stress-strain curve of the material after optimization
Table 3 Characteristic parameter value after optin

\begin{tabular}{ccccc} 
Parameter & $\mathrm{m}_{1}$ & $\mathrm{~m}_{2}$ & $\mathrm{~m}_{3}$ & $\mathrm{~m}_{4}$ \\
\hline Value & 0.432 & 3.175 & 6.132 & 32.432 \\
Paremeter & $\mathrm{n}_{1}$ & $\mathrm{n}_{2}$ & $\mathrm{n}_{3}$ & $\mathrm{n}_{4}$ \\
Value & 0.32 & 0.31 & 0.24 & 0.13 \\
\hline
\end{tabular}

\section{Conclusions}

This paper mainly studies the vehicle collision simulation dummy material parameter optimization design method. Parameterize parametric curves and optimization curves, and let these parameters represent the main features of the curve, The optimization problem of complex curve to curve is transformed into multi parameter and multi objective optimization problem. At the same time, the multi objective optimization problem is piecewise linear, make the problem easier to calculate. Use this method to optimize result curve in the process of development of dummy is important. This method is further verified in a numerical example. In conclusion, The optimum design method can be used for dummy experiment curve optimization research in vehicle collision .

Table 4 Evaluation indexes of thoracic pressure curve after optimization

\begin{tabular}{cc|c|c}
\hline $\begin{array}{c}\text { valuation indexes } \\
\text { Value }\end{array}$ & $\mathrm{D}_{\max } /(\mathrm{mm})$ & $\mathrm{K}_{1}$ & $\mathrm{~K}_{2}$ \\
& 30.089 & -2.505 & 2.105 \\
\hline
\end{tabular}

\section{Acknowledgements}

This work was financially supported by the Automotive parts manufacturing technology and equipment, Jilin province colleges and universities high end science and technology innovation platform (440020031101).

\section{References}

[1] ZHAO Fuquan, WU Chengming, PAN Zhijie,et al. Journal of Automotive Safety and Energy,2011,2(2):111-121 In Chinese.

[2] Cing-Dao(steve)Kan, Dhafer Marzougui, Nabih E. Bedewi. Development of a 50th Percentile Hybrid III Dummy Model[C].4th European LS-DYNA Users Conference, 2006:4-5.

[3] FTSS(First Technology Safely Systems).For Immediate Release. [EB/OL] ,2008.

[4] Z.Zhou, M.Li, J.Rasico, F.Zhu, R.kant (FTSS, Inc.). A Full Suite of Hybrid III 50th Dummy Models with the Latest upgrades-from Savor,High Quality Performer, to the More Detailed 
Model (E). LS-DYNA Anwenderforum, Frankenthal 2007,18-22.

[5] Ruan Jesse, EI-Jawahri Raed;Li Chai, et al. Prediction and analysis of human thoracic impact responses and injuries in cadaver impacts using a full human body finite element model. Stapp Car Crash Journal, 2003. 47: 299-321.

[6] Noureddine, A., A. Eskandarian, and K. Digges, Computer modeling and validation of a hybrid III dummy for crashworthiness simulation. Mathematical and Computer Modelling, 2002. 35(7-8): p. 885-893.

[7] Robert A.Denton,Inc. History of Anthropomorphic Test Devices,2008,1-3.

[8]TNO.MADYMO Model Manual Version 6.2.1.Holland:Road Vehicle Resea-rch Institute,2004,23-48

[9] CHEN Shuang,YUAN Zhong-fan,LIN Da-quan,et al. Journal of Sichuan University,2008,40(3):178-182 In Chinese.

[10] MA Kai1,GUAN Hsin,PANG Shu-yi,et al. Journal of Jilin University,2011,41(4):910-914 In Chinese. 